\title{
ACTA Urológica Portuguesa
}

www.elsevier.pt/acup

CASO CLÍNICO

\section{Obstrução da junção pieloureteral por pólipos fibroepiteliais em idade pediátrica}

\section{Maria Knoblich*, Vanda Pratas Vital, Dinorah Cardoso, Fátima Alves, Filipe Catela Mota e Paolo Casella}

Serviço de Cirurgia Pediátrica, Unidade de Urologia Pediátrica, Hospital Dona Estefânia, Centro Hospitalar de Lisboa Central, Lisboa, Portugal

Recebido a 9 de abril de 2014; aceite a 10 de outubro de 2016

Disponível na Internet a 2 de novembro de 2016

\section{PALAVRAS-CHAVE \\ Pólipos \\ fibroepiteliais; \\ Obstrução da junção \\ pieloureteral; \\ Hidronefrose; \\ Tumores benignos}

\section{KEYWORDS}

Fibroepithelial

polyps;

Ureteropelvic

junction obstruction;

Hydronephrosis;

Benign tumors

\begin{abstract}
Resumo
Introdução: Os pólipos fibroepiteliais do ureter são tumores benignos de origem mesodérmica extremamente raros em idade pediátrica, que podem causar obstrução da junção pieloureteral (JPU).

Caso clínico: Os autores apresentam o caso de um doente do sexo masculino, de 11 anos de idade, referenciado por dor no flanco esquerdo intermitente associada a hidronefrose esquerda. Intraoperatoriamente, constatou-se a obstrução do lúmen ureteral provocada pela presença de pólipos fibroepiteliais ao nível da JPU.

Discussão: 0 tratamento standard consiste na ressecção do segmento ureteral afetado, seguida de pieloplastia desmembrada, por via aberta ou laparoscópica. O prognóstico é excelente.

Publicado por Elsevier España, S.L.U. em nome de Associação Portuguesa de Urologia. Este é um artigo Open Access sob uma licença CC BY-NC-ND (http: //creativecommons.org/licenses/ by-nc-nd/4.0/).
\end{abstract}

\section{Ureteropelvic junction obstruction caused by Fibroepithelial polyps in children}

\section{Abstract}

Introduction: Fibroepithelial polyps are extremely rare benign tumors arising from mesodermal tissue in the ureteral wall in children, that can cause ureteropelvic junction obstruction. Case report: In this report, we present an 11-year-old boy complaining of left intermitent lumbar pain related with ipsilateral hydronephrosis. Surgical exploration revealed several endoluminal polyps located at the ureteropelvic junction, obstructing the lumen of the ureter.

\footnotetext{
* Autor para correspondência.

Correio eletrónico: mknoblich@gmail.com (M. Knoblich).
} 
Discussion: Standard treatment consists in resection of the affected ureteral segment followed by open or laparoscopic dismembered pyeloplasty. The prognosis is excellent.

Published by Elsevier España, S.L.U. on behalf of Associação Portuguesa de Urologia. This is an open access article under the CC BY-NC-ND license (http://creativecommons.org/licenses/bync-nd/4.0/).

\section{Introdução}

A hidronefrose secundária a obstrução da junção pieloureteral (JPU) é comum em idade pediátrica. É maioritariamente causada por fatores congénitos, intrínsecos ou extrínsecos, como a estenose da JPU, vasos polares inferiores anómalos, bandas fibróticas, válvulas papilares, etc. Adicionalmente, pode resultar de fatores adquiridos como cálculos ou coágulos. Os pólipos fibroepiteliais representam apenas 0,5\% das obstruções pieloureterais que requerem pieloplastia em crianças $^{1}$, devendo, no entanto, ser uma causa equacionada em doentes com dor abdominal ou lombar intermitente e hidronefrose. Os autores apresentam o caso clínico de uma criança com obstrução da JPU provocada por pólipos fibroepiteliais, cuja suspeita pré-operatória era de etiologia obstrutiva, por presença de vaso polar inferior anómalo.

\section{Caso clínico}

Doente do sexo masculino com 11 anos de idade, referenciado ao serviço de urgência por dor lombar esquerda, tipo cólica, acompanhada de vómitos. Apresentava história de lombalgia esquerda recorrente e diagnóstico ecográfico de dilatação pielocalicial esquerda desde os 6 anos, altura em que foi referenciado à consulta de nefrologia, à qual não compareceu.

Ao exame objetivo constatou-se sinal de Murphy renal à esquerda positivo. Analiticamente, a análise sumária de urina revelou leucocitúria e a urocultura foi negativa.

A ecografia renal documentou hidronefrose esquerda, com um bacinete de diâmetro máximo de $40 \mathrm{~mm}$, coexistindo ectasia dos grupos caliciais, espessura do parênquima renal globalmente conservada, mas com diminuição da diferenciação corticomedular, sem visualização do ureter homolateral.

A urografia endovenosa mostrou uma imagem de subtração transversal ao nível da JPU (fig. 1), confirmada por tomografia axial computorizada (TC), com fase excretora, revelou vasos polares inferiores e obstrução ao nível da JPU esquerda sem défice funcional significativo, uma vez que a excreção ocorreu num tempo precoce.

Ambos os exames de imagem pareciam apontar para uma obstrução da JPU por um vaso anómalo polar inferior, carecendo de confirmação por angio-TC.

0 doente foi proposto para pieloplastia desmembrada aberta. Intraoperatoriamente, constatou-se a existência de obstrução intrínseca da JPU pela presença de 2 pólipos ao nível do ureter proximal (figs. 2 e 3 ). Foi efetuada pieloplastia desmembrada segundo a técnica de Anderson-Hynes

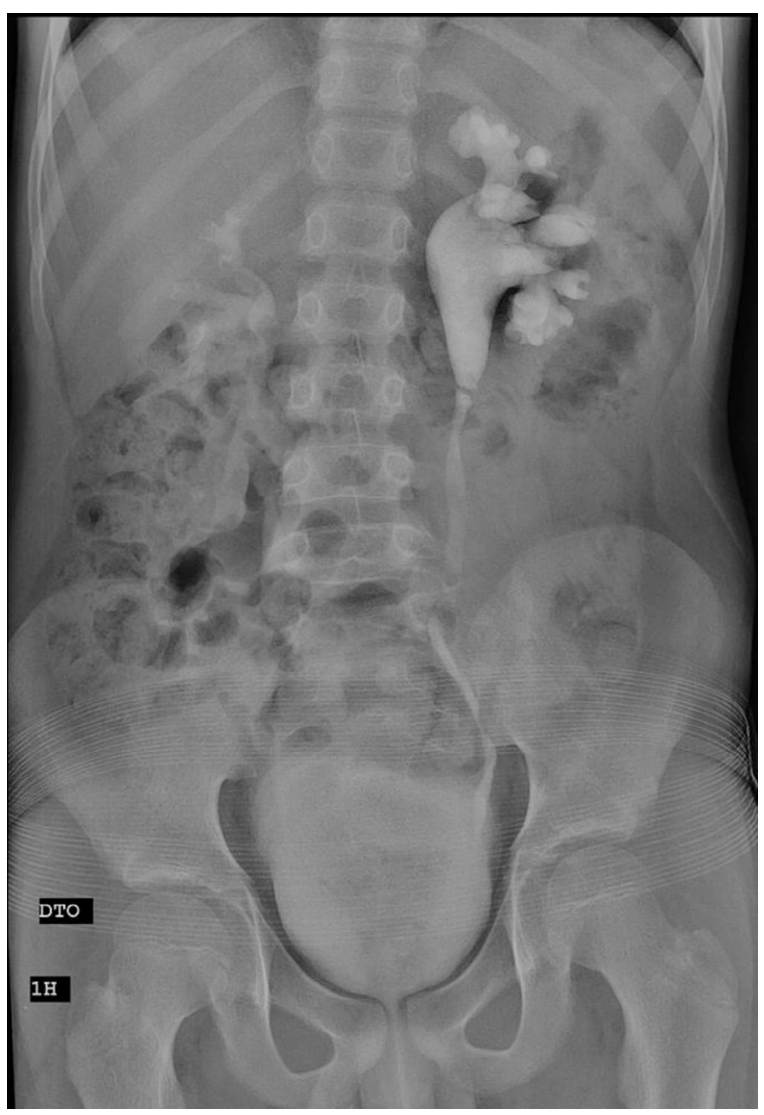

Figura 1 Urografia endovenosa mostra uma imagem de subtração transversal ao nível da JPU.

sobre tutor ureteral duplo J, após remoção da JPU e de segmento do ureter proximal.

0 pós-operatório imediato decorreu sem intercorrências. A remoção endoscópica do stent ocorreu 4 meses depois.

O exame anatomopatológico confirmou o diagnóstico de pólipos fibroepiteliais com ligeiro infiltrado inflamatório linfocitário da mucosa e ligeira hiperplasia da camada muscular com fibrose intersticial.

Um ano após o procedimento a criança mantinha-se assintomática e a ecografia renovesical de controlo não mostrava hidronefrose significativa.

\section{Discussão}

Os pólipos fibroepiteliais são os tumores benignos mais comuns do ureter. São tumores raros de origem 


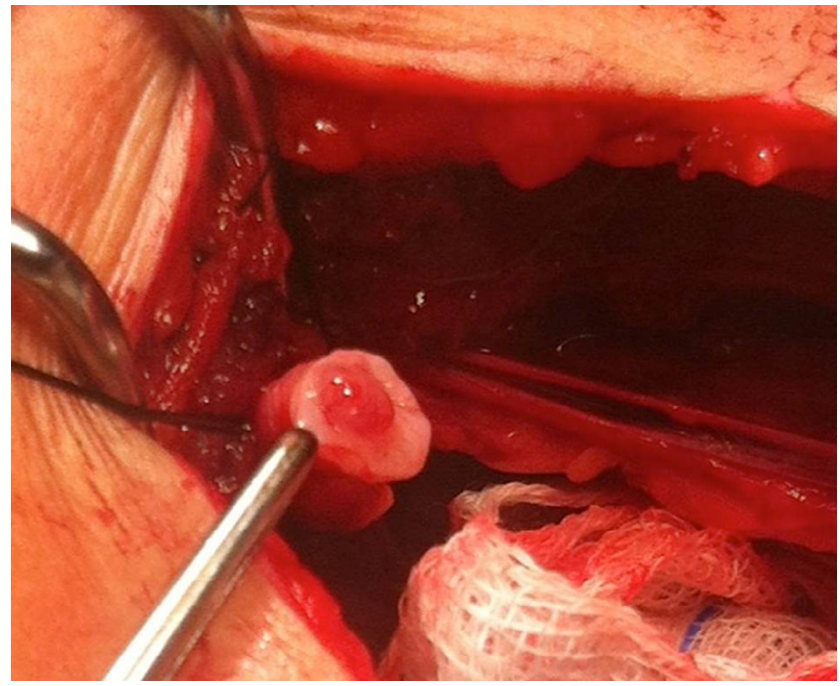

Figura 2 Imagem intraoperatória de pólipo do ureter proximal.

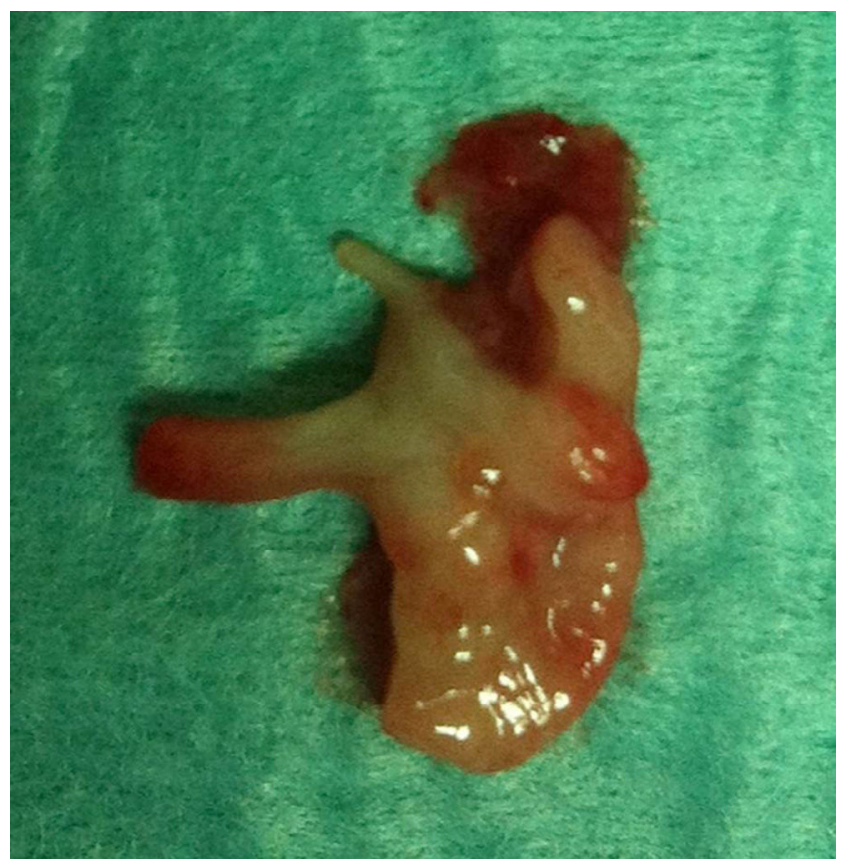

Figura 3 Peça operatória. A seta indica um pólipo intraureteral.

mesodérmica, que correspondem a projeções da mucosa compostas por estroma fibroso coberto por urotélio ${ }^{2}$, que podem ocorrer em todo o aparelho urinário. A localização mais comum destes pólipos é a JPU e o ureter proximal, seguidos da uretra posterior e do ureter médio e distal ${ }^{3}$. Outros tumores benignos menos comuns são os leiomiomas, papilomas, hemangiomas, linfangiomas, granulomas ou fibromas. São mais frequentes no sexo masculino e do lado esquerdo $^{3-5}$. Podem ser únicos ou, mais frequentemente, múltiplos ${ }^{6}$.

Em adultos, a maioria dos tumores epiteliais do ureter são malignos. No entanto, em idade pediátrica os tumores malignos são muito raros.
A etiologia dos pólipos é desconhecida. Existem várias causas apontadas, entre as quais, inflamação ou infeção crónica, obstrução, trauma e alterações endógenas e hormonais $^{7,8}$.

Os doentes geralmente apresentam dor lombar ou abdominal pela hidronefrose obstrutiva. A maioria refere episódios de cólica renal. Em alguns casos pode ocorrer hematúria ou outros sintomas sugestivos de infeção do aparelho urinário .

O diagnóstico pré-operatório de obstrução da JPU por pólipo é feito apenas em cerca de $22 \%$ dos doentes ${ }^{1}$.

A ecografia pode revelar uma hidronefrose moderada a severa, sem dilatação do ureter, mostrando uma estrutura ecogénica com projeção polipoide estendendo-se para o ureter, sem sombra acústica. Sendo difícil o diagnóstico pré-operatório de obstrução da JPU por pólipo, deve existir um elevado índice de suspeição durante a realização de ecografia, nomeadamente na visualização de vascularização intraluminal por Doppler.

A urografia endovenosa pode revelar um defeito de preenchimento na JPU ou no lúmen ureteral, que pode ser confundido com uma prega da mucosa. A ecografia tem uma acuidade diagnóstica superior à urografia endovenosa e deve ser a primeira escolha no diagnóstico de pólipos fibroepiteliais na criança ${ }^{5,10}$. A TC pode revelar preenchimento de tecido mole na JPU e ureter proximal ${ }^{10}$. Em fase excretora com contraste não acrescenta mais informação do que a urografia endovenosa ${ }^{7}$.

A obstrução completa da JPU a longo prazo pode levar a atrofia cortical e, consequentemente, a uma perda de função renal, e nestes doentes é necessário a realização de cintigrafia renal para avaliar a função renal. Daí que seja necessário vigiar a função renal e os sintomas dos doentes com hidronefrose, para diferenciar aqueles que podem manter-se em vigilância dos que requerem intervenção. 0 tratamento adequado consiste na ressecção segmentar do ureter, contendo os pólipos e na realização de pieloplastia desmembrada de Anderson-Hynes, quer por técnica aberta quer por via laparoscópica ${ }^{1,9,11}$. A ressecção dos pólipos por ureterorrenoscopia é uma opção minimamente invasiva nas crianças, mas a ressecção do pólipo pode ser incompleta, aumentando a probabilidade de recorrência ${ }^{12}$. Com a ressecção segmentar e a pieloplastia desmembrada, o prognóstico dos pólipos fibroepiteliais é excelente. Não há registo de recorrência dos casos descritos na literatura, neste tipo de abordagem ${ }^{3}$.

\section{Responsabilidades éticas}

Proteção de pessoas e animais. Os autores declaram que para esta investigação não se realizaram experiências em seres humanos e/ou animais.

Confidencialidade dos dados. Os autores declaram ter seguido os protocolos do seu centro de trabalho acerca da publicação dos dados de pacientes.

Direito à privacidade e consentimento escrito. Os autores declaram que não aparecem dados de pacientes neste artigo. 


\section{Conflito de interesses}

\section{Os autores declaram não haver conflito de interesses.}

\section{Bibliografia}

1. Adey GS, Vargas SO, Retik AB, Borer JC, Mandell J, Hendren WH, et al. Fibroepithelial polyps causing ureteropelvic junction obstruction in children. J Urol. 2003;169:1834-6.

2. Stuppler SA, Kandzari SJ. Fibroepithelial polyps of the ureter. A benign ureteral tumor. Urology. 1975;5:553-8.

3. Musselman P, Kay R. The spectrum of urinary tract fibroepithelial polyps in children. J Urol. 1986;136:476-7.

4. Galifer RB, Aubert D, Couture A, Veyrac C, Barneon C, Kötter R. Fibrous epithelial polyps of the pyelo-ureteral junction in children. Presentation of 3 cases and review of the literature (33 cases). Chir Pediatr. 1988;29:318-24.

5. Niu ZB, Yang Y, Hou Y. Ureteral polyps: An etiological factor of hydronephrosis in children that should not be ignored. Pediatr Surg Int. 2007;23:323-6.
6. Karaca I, Sencan A, Mir E, Sayan A, Ortaç R. Ureteral fibroepithelial polyps in children. Pediatr Surg Int. 1997;12:603-4.

7. Casser Delia E, Joseph VT, Sherwood W. Fibroepithelial polyps causing ureteropelvic junction obstruction in children - A case report and review article. Eur J Pediatric Surg. 2007;17:142-6.

8. Li WM, Wu WJ, Ke HL, Chai CY, Chou YH, Huang CH. Fibroepithelial polyps causing ureteropelvic junction obstruction in a child. Kaohsiung J Med Sci. 2005;21:282-5.

9. Macksood MJ, Roth DR, Chang $\mathrm{CH}$, Perlmutter AD. Benign fibroepithelial polyps as a cause of intermittent ureteropelvic junction obstruction in a child: A case report and review of the literature. J Urol. 1985;134:951-2.

10. Wang $X M$, Jia LQ, Wang $Y$, Wang $N$. Utilizing ultrasonography in the diagnosis of pediatric fibroepithelial polyps causing ureteropelvic junction obstruction. Pediatr Radiol. 2012;42:1107-11.

11. Iwatsuki S, Kojima Y, Mizuno K, Tozawa K, Kohri K, Hayashi Y. Laparoscopic management for fibroepithelial polyp causing ureteropelvic junction obstruction in a child. J Urology. 2009, 12.062 .

12. Sharma NK, Stephenson RN, Tolley DA. Endoscopic management of fibroepithelial polyps in the ureter. Br J Urol. 1996;78:131-2. 